\title{
The Integrated Density of States of the Random Graph Laplacian
}

\author{
T. Aspelmeier • A. Zippelius
}

Received: 31 December 2010 / Accepted: 7 July 2011 / Published online: 29 July 2011

(C) The Author(s) 2011. This article is published with open access at Springerlink.com

\begin{abstract}
We analyse the density of states of the random graph Laplacian in the percolating regime. A symmetry argument and knowledge of the density of states in the nonpercolating regime allows us to isolate the density of states of the percolating cluster (DSPC) alone, thereby eliminating trivially localised states due to finite subgraphs. We derive a nonlinear integral equation for the integrated DSPC and solve it with a population dynamics algorithm. We discuss the possible existence of a mobility edge and give strong evidence for the existence of discrete eigenvalues in the whole range of the spectrum.
\end{abstract}

Keywords Random graphs · Graph Laplacian · Density of states

\section{Introduction}

The eigenvalue spectrum of sparse random matrices is a fascinating and largely unsolved problem with widespread applications ranging from transport in disordered systems, graph theory and optimisation problems to nuclear physics and QCD [1, 2]. In this paper we consider a prototype of such a random matrix, the Laplace operator on a mean-field random graph with $N$ nodes, i.e. a graph where a link between two arbitrary sites is either present with probability $p=2 c / N$ or not present with probability $1-p$. The constant $2 c$ is the mean connectivity of the graph. The Laplace operator on this graph is a matrix $\Gamma_{i j}$ where for $i \neq j \Gamma_{i j}=-1$ if the nodes $i$ and $j$ of the graph are connected and $\Gamma_{i j}=0$ otherwise,

T. Aspelmeier $(\bowtie) \cdot$ A. Zippelius

Max Planck Institut für Dynamik und Selbstorganisation, Bunsenstr. 10, 37073 Göttingen, Germany

e-mail: aspe1@ theorie.physik.uni-goettingen.de

T. Aspelmeier

Scivis GmbH, Bertha-von-Suttner-Str. 5, 37085 Göttingen, Germany

\section{A. Zippelius}

Institut für Theoretische Physik, Georg-August Universität Göttingen, Friedrich-Hund-Platz 1, 37077

Göttingen, Germany 
while on the diagonal $\Gamma_{i i}=-\sum_{j \neq i} \Gamma_{i j}$. The entries on the diagonal are thus correlated to the random entries outside the diagonal.

Even though the computation of the density of states for a mean-field random graph has been reduced to an integral equation [3-5], a complete solution is still missing. In the limit of infinite coordination, $c \rightarrow \infty$, Wigner's semi-circle law is recovered. For any finite $c$, Lifshitz tails were shown to exist in the integrated density of states [6]. Beyond these asymptotic results a number of approximations have been used to compute the spectrum approximately, such as effective medium theory [7], single defect approximation [8], moment expansions, numerical diagonalisation [9, 10] and regularisation techniques [9].

In this paper we are mainly interested in the density of states of the percolating cluster. This problem is a special case of the spectral theory of random operators on percolation subgraphs (see [11] for a review). Here, we show first that the density of states of the percolating cluster (DSPC) can be isolated using a symmetry argument and our knowledge of the density of states below the percolation threshold. Second, we derive an integral equation for the integrated density of states which can be solved reliably with a population algorithm. The numerical solution reveals jumps in the integrated DSPC in the whole range of the spectrum, calling in question the existence of a mobility edge. We are also able to find the asymptotic behaviour of the integrated DSPC of the incipient percolating cluster at the percolation threshold for small eigenvalues.

\section{Model and Symmetry}

The model shows a percolation transition at $c_{\text {crit }}=1 / 2$. Below this concentration there is no macroscopic cluster and almost all finite clusters are trees. The average number of tree clusters $T_{n}$ with $n$ nodes is given in the macroscopic limit by [12]

$$
\lim _{N \rightarrow \infty} \frac{T_{n}(2 c)}{N}=\tau_{n}(2 c)=\frac{n^{n-2}\left(2 c e^{-2 c}\right)^{n}}{2 c n !} .
$$

In particular the average total number of clusters per particle is $\tau_{\text {tot }}(2 c)=1-c$. For $c<1 / 2$, the spectrum consists of a very complicated, but countable set of $\delta$-peaks which can be calculated iteratively [10]. Above the percolation threshold $c>1 / 2$ a percolating cluster coexists with many finite clusters, almost all of which are also trees. The fraction of sites in the macroscopic cluster, $Q(c)$, is the solution of $1-Q(c)=\exp (-2 c Q(c))$ [12]. Alternatively we rewrite $Q(c)=1-\frac{x(c)}{2 c}$ and obtain $x(c)$ as the solution of

$$
x(c) e^{-x(c)}=2 c e^{-2 c} .
$$

This equation has two solutions, a trivial one with $x(c)=2 c$ and a nontrivial one with $x(c)=2 c^{*}$ such that $c^{*}>\frac{1}{2}$ if $c<\frac{1}{2}$ and vice versa. This nontrivial solution allows one to establish a symmetry for the number of trees with a fixed number of nodes above and below the percolation threshold. Using (2), we can rewrite (1) according to

$$
\tau_{n}(x(c))=\frac{2 c}{x(c)} \tau_{n}(2 c) \quad \text { or } \quad \tau_{n}\left(2 c^{*}\right)=\frac{c}{c^{*}} \tau_{n}(2 c) .
$$

Hence the number of trees above the percolation threshold is simply related to the number of trees below the percolation threshold. 


\section{Density of States}

This relation allows us to compute the density of states of the percolating cluster alone, which is the quantity of primary interest. It is known that the density of states, $D(\Omega)$, of the infinite cluster contains at least some $\delta$ peaks, so that a population dynamics algorithm [9] cannot be applied. In this paper we instead compute the integrated density of states, $\Delta(\Omega)=$ $\int_{0}^{\Omega} d \Omega^{\prime} D\left(\Omega^{\prime}\right)$, which according to measure theory [13], may be decomposed into a singular part and an absolutely continuous part. The absolutely continuous part may itself consist of two contributions, eigenvalues stemming from localised eigenvectors that lie dense and each have vanishing weight in the thermodynamic limit, and the eigenvalues stemming from nonlocalised eigenvectors. We may thus write

$$
\Delta(\Omega)=\Delta_{\text {loc, } \operatorname{disc}}(\Omega)+\Delta_{\text {loc, cont }}(\Omega)+\Delta_{\text {nonloc }}(\Omega) .
$$

Often in random matrix problems there is a mobility edge, i.e. a value $\Omega_{0}$ such that all eigenvectors corresponding to eigenvalues $\Omega<\Omega_{0}$ are localised and all eigenvectors corresponding to $\Omega>\Omega_{0}$ are nonlocalised (or vice versa). We will show here that such a sharp edge does not seem to exist in our problem as we find discrete eigenvalues even in the region where nonlocalised eigenvectors lie. This shows that a naive approach using the inverse participation ratio in order to find the onset of nonlocalised eigenvectors will not work since the localised eigenvectors do not disappear where the nonlocalised ones first appear, so the inverse participation ratio will not drop down to 0 as it would at a true mobility edge.

The density of eigenvalues, $\left\{\Omega_{i}\right\}_{i=1}^{N}$, of the Laplacian matrix $\Gamma$ is defined by

$$
D(\Omega, c)=\lim _{N \rightarrow \infty} \overline{\frac{1}{N} \sum_{i=1}^{N} \delta\left(\Omega-\Omega_{i}\right)}=\lim _{N \rightarrow \infty} \overline{\frac{1}{N} \operatorname{Tr} \delta(\Omega-\Gamma)} .
$$

Here - denotes the average over all realisations of connectivity for a given $c$. To compute the density of eigenvalues we introduce the resolvent

$$
G(\Omega, c)=\lim _{N \rightarrow \infty} \frac{1}{N} \overline{\operatorname{Tr} \frac{1}{\Gamma-\Omega}}
$$

for complex argument $\Omega=\gamma+i \epsilon, \epsilon>0$. In the limit $\epsilon \rightarrow 0$, we recover the spectrum from the imaginary part of the resolvent according to

$$
D(\Omega, c)=\frac{1}{\pi} \lim _{\epsilon \downarrow 0} \Im G(\Omega+i \epsilon, c) .
$$

In the percolating regime $c \geq \frac{1}{2}$ the macroscopic cluster coexists with many finite ones. In order to study localised states of the macroscopic cluster it is essential to isolate the density of states of the macroscopic cluster only. The symmetry expressed by (2) and (3) allows us to decompose the resolvent into two contributions, one from the percolating cluster and one from the finite clusters. Since the finite clusters from $c^{*}$ are also present at $c$ (although with a smaller total weight), we write $G(\Omega, c)$ as a sum of the resolvent $G\left(\Omega, c^{*}\right)$, appropriately weighted, and a remainder $G^{\text {perc }}(\Omega, c)$, which then must generate the spectrum of the giant cluster:

$$
G(\Omega, c)=G^{\mathrm{perc}}(\Omega, c)+\frac{c^{*}}{c} G\left(\Omega, c^{*}\right)
$$


The above relation allows us to compute the density of states of the percolating cluster alone from the full density of states above, $G(\Omega, c)$, and the density of states below the percolation threshold, $G\left(\Omega, c^{*}\right)$ for $c^{*}<\frac{1}{2}$, which is known [10].

The resolvent can be expressed in terms of an auxiliary function $g_{c, \Omega}(\rho)$ as [3]

$$
G(\Omega, c)=-1+\frac{i}{2 c} \int_{0}^{\infty} d \rho \rho g_{c, \Omega}(\rho) .
$$

The average over all realisations of connectivity is performed with the replica trick, resulting in a nonlinear integral equation for $g_{c, \Omega}(\rho)$ (cf. (16) and (17) in Ref. [3])

$$
\begin{aligned}
g_{c, \Omega}(\rho)= & 2 c \exp \left\{-\frac{i \rho^{2}}{2}\right\} \\
& +2 i c e^{-2 c} \int_{0}^{\infty} d x \rho I_{1}(i \rho x) \exp \left\{-\frac{i}{2}\left(\rho^{2}+x^{2}\right)+\frac{i \Omega}{2} x^{2}+g_{c, \Omega}(x)\right\}
\end{aligned}
$$

with $g_{c, \Omega}(0)=2 c$. Here $I_{v}(z)$ are the modified Bessel functions of the first kind.

\subsection{Nonpercolating Regime}

Equation (10) for $c<1 / 2$ was solved in [10]. The solution is

$$
g_{c, \Omega}(\rho)=2 c \sum_{k} a_{k} \exp \left(-\frac{i}{2} z_{k} \rho^{2}\right)=2 c \int_{-\infty}^{\infty} d a_{c, \Omega}(\lambda) e^{-\frac{i}{2} \lambda \rho^{2}}
$$

with coefficients $a_{k}$ and $z_{k}$ to be defined below. The sum can also be expressed as a RiemannStieltjes integral with weight function $a_{c, \Omega}(\lambda)=\sum_{k} a_{k} \theta\left(\lambda-z_{k}\right)(\theta(\cdots)$ is the Heaviside function). This formulation will be useful later.

The coefficients $a_{k}$ and $z_{k}$ can be grouped in infinitely many "classes." The coefficients of class $n+1$ are given recursively by the relations

$$
\begin{aligned}
& a_{\left(l_{k}\right)}^{(n+1)}=e^{-2 c} \prod_{k} \frac{\left(2 c a_{k}^{(n)}\right)^{l_{k}}}{l_{k} !} \\
& z_{\left(l_{k}\right)}^{(n+1)}=\frac{\Omega-\sum_{k} l_{k} z_{k}^{(n)}}{\Omega-1-\sum_{k} l_{k} z_{k}^{(n)}}
\end{aligned}
$$

(the upper index denotes the class). Class 0 contains only one element, namely $a_{0}^{(0)}=e^{-2 c}$ and $z_{0}^{(0)}=\frac{\Omega}{\Omega-1}$. The index on the left hand side is a finite sequence $\left(l_{k}\right)$ of nonnegative integers. In order to proceed to the next stage of the recursion, $\left(l_{k}\right)$ must be mapped to a natural number $m$ since for the calculation of, say, $z_{\left(l_{k}\right)}^{(n+1)}$ it is necessary to access the coefficients $z_{m}^{(n)}$ of the previous iteration. Such a mapping is possible because the set of sequences $\left\{\left(l_{k}\right)\right\}$ is countably infinite. It was shown in [10] that the correct way to do the mapping is to choose $m=\sum_{k} l_{k} M^{k}$ and to let $M$ (formally) tend to infinity at an appropriate point. Note that class $n+1$ also contains all coefficients from class $n$. See [10] for details.

These classes give us an infinite hierarchy of coefficients, each recursion step adding infinitely many coefficients to the previous ones. Note that the coefficients in class $n$ constructed in this way are not an approximation but constitute (part of) the exact solution of (10). Only the total weight of coefficients $\sum_{k} a_{k}$ falls short of 1 when stopping the recursion at a finite $n$. The complete sets of coefficients $a_{k}$ and $z_{k}$ from (11) are the collection 
of all coefficients from all classes, i.e. the are equal to $a_{l_{k}}^{(\infty)}$ and $z_{l_{k}}^{(\infty)}$ from the recursion, appropriately reindexed. It follows from this representation that for any finite sequence of nonnegative integers $\left(l_{k}\right)$ there exists an index $k^{\prime}$ such that

$$
\begin{aligned}
& e^{-2 c} \prod_{k} \frac{\left(2 c a_{k}\right)^{l_{k}}}{l_{k} !}=a_{k^{\prime}} \quad \text { and } \\
& \frac{\Omega-\sum_{k} l_{k} z_{k}}{\Omega-1-\sum_{k} l_{k} z_{k}}=z_{k^{\prime}} .
\end{aligned}
$$

This will be useful below.

The solution of (10) which is given in (11) leads to a density of states consisting of $\delta$ peaks which are located at those $\Omega$ where $z_{k}=0$.

\subsection{Percolating Regime}

In analogy to the resolvent, we can decompose $g_{c, \Omega}(\rho)=g_{c, \Omega}^{\text {perc }}(\rho)+g_{c^{*}, \Omega}(\rho)$ into a part $g_{c, \Omega}^{\text {perc }}(\rho)$ pertaining to the infinite cluster and a part which is equal to the (known) solution $g_{c^{*}, \Omega}(\rho)$ of the integral equation at $c^{*}$. This decomposition has the advantage that we can directly obtain the density of states of the infinite cluster by deriving an equation for $g_{c, \Omega}^{\text {perc }}(\rho)$, which in analogy to (11) can be represented as

$$
g_{c, \Omega}^{\text {perc }}(\rho)=\left(2 c-2 c^{*}\right) \int_{-\infty}^{\infty} d b_{c, \Omega}(\lambda) e^{-\frac{i}{2} \lambda \rho^{2}} .
$$

The function $b_{c, \Omega}(\lambda)$ is normalised such that $\int_{-\infty}^{\infty} d b_{c, \Omega}(\lambda)=1$ and the "initial condition" $g_{c, \Omega}(0)=2 c$ is being taken care of by the prefactor $2 c-2 c^{*}$. This ansatz is plugged into the integral (10) and yields, after application of (14) and (15),

$$
\begin{aligned}
b_{c, \Omega}(\lambda)= & 2 c^{*} \sum_{n=0}^{\infty} a_{n} \sum_{M=1}^{\infty} \frac{\left(2 c-2 c^{*}\right)^{M-1}}{M !} \int_{-\infty}^{\infty} d b_{c, \Omega}\left(\lambda_{1}\right) \cdots \int_{-\infty}^{\infty} d b_{c, \Omega}\left(\lambda_{M}\right) \\
& \times \theta\left(\lambda-\left[1-\frac{1}{\frac{1}{1-z_{n}}+\sum_{i=1}^{M} \lambda_{i}}\right]\right)
\end{aligned}
$$

This equation can be solved numerically by running a population dynamics algorithm for the coefficients $z_{k}$ at $c^{*}$ below the critical point in parallel to a population dynamics for a $\lambda$-population at $c$ above the critical point, for which the $z_{k}$ and their weights $a_{k}$ are needed as input.

The population dynamics algorithm below the critical point consists of keeping a large population of $z_{k}$ 's and repeatedly updating this population by choosing a Poisson random number $n$ with parameter $2 c^{*}$, randomly selecting $n$ of the $z_{k}$ with indices $k_{i}(i=1, \ldots, n)$ and inserting $1+\frac{1}{\Omega-1-\sum_{i=1}^{n} z_{k_{i}}}$ as a new member into the population, replacing a random existing member. This process yields a numerical approximation of the distribution $a_{c^{*}, \Omega}(\lambda)$.

The population dynamics above the critical point is similar. A large $\lambda$ population is updated by first drawing a Poisson random number $M$ with parameter $2 c-2 c^{*}$, but rejecting and redrawing it if it is 0 (this step corresponds to the sum over $M$ in (17) which starts with $M=1$, and which is correctly normalised for this modified Poisson distribution). If $c=c^{*}$, this rule does not work and (17) shows that in this case $M=1$ must be chosen with 
probability 1 . Then some $\lambda_{i}(i=1, \ldots, M)$ are drawn randomly from the $\lambda$ population, and one $z$ is drawn randomly from the population of the $z_{k}$ calculated above. Finally the new member $1-\frac{1}{\frac{1}{1-z}+\sum_{i=1}^{M} \lambda_{i}}$ replaces a random existing $\lambda$. This is repeated until convergence (in a statistical sense) is achieved. The $\lambda$ population then approximates $b_{c, \Omega}(\lambda)$.

Given $a_{c^{*}, \Omega}(\lambda)$ and $b_{c, \Omega}(\lambda)$ the integrated DSPC can be computed according to (see Appendix A):

$$
\begin{aligned}
& 2 \Delta^{\mathrm{perc}}(\Omega, c) \\
& =1+\int_{-\infty}^{\infty} d b_{c, \Omega}(\lambda)\left(\operatorname{sgn}\left(\frac{\lambda}{\lambda-1}\right)+c \operatorname{sgn}(\lambda-1)\right) \\
& \quad-\left(c-c^{*}\right) \int_{-\infty}^{\infty} d b_{c, \Omega}(\lambda) d b_{c, \Omega}\left(\lambda^{\prime}\right) \operatorname{sgn}\left(\frac{\lambda}{\lambda-1}-\lambda^{\prime}\right) \\
& \quad+c^{*} \int d a_{c^{*}, \Omega}(\lambda) \operatorname{sgn}\left(\frac{1}{\lambda-1}\right) \\
& \quad-c^{*} \int\left(d a_{c^{*}, \Omega}(\lambda) d b_{c, \Omega}\left(\lambda^{\prime}\right)+d b_{c, \Omega}(\lambda) d a_{c^{*}, \Omega}\left(\lambda^{\prime}\right)\right) \operatorname{sgn}\left(\frac{\lambda}{\lambda-1}-\lambda^{\prime}\right)
\end{aligned}
$$

\section{Discussion}

We have developed a systematic approach to compute the integrated DSPC. We stress that the (nonintegrated) DSPC cannot easily be obtained from a conventional population dynamics algorithm without regularisation methods, which was done in [9]. Naively it is given in terms of $b_{c, \Omega}(\lambda)$ by

$$
\begin{aligned}
D^{\text {perc }}(\Omega, c) & =\frac{1}{\pi} \Im \int_{0}^{\infty} d \rho \rho \int_{-\infty}^{\infty} d b_{c, \Omega}(\lambda) e^{-\frac{i}{2} \lambda \rho^{2}} \\
& =\int_{-\infty}^{\infty} d b_{c, \Omega}(\lambda) \delta(\lambda) .
\end{aligned}
$$

The above density of states would be very simple if $b_{c, \Omega}(\lambda)$ was differentiable with respect to $\lambda$, as it would then be equal to $\left(b_{c, \Omega}\right)^{\prime}(0)$. It is however known that the density of states of the percolating cluster contains $\delta$ peaks also for $c>1 / 2$ [14]. Hence the position and weight of the $\delta$ peaks cannot directly be obtained from a population dynamics algorithm-a problem already encountered in the nonpercolating regime. It is thus essential to either use regularisation as in [9] or to obtain the integrated density of states directly from population dynamics without going to the density of states first and integrating, and this is what (17) and (18) provide.

The final equation for the integrated DSPC, (18), reveals much about the eigenvalues of the percolating cluster. We can, for example, understand the origin of the $\delta$ peaks in the density of states of the percolating cluster. Suppose for the moment that $b_{c, \Omega}(\lambda)$ is continuous as a function of $\Omega$. Then the integrals over $d b_{c, \Omega}(\lambda)$ certainly do not generate any jumps in $\Delta^{\text {perc }}(\Omega, c)$ due to the continuity in $\Omega$. However, we know that $a_{c^{*}, \Omega}(\lambda)=\sum_{n} a_{n} \theta\left(\lambda-z_{n}\right)$ is discontinuous as a function of $\Omega$ since the $z_{n}$ depend on $\Omega$, and inspection of (18) shows that this leads to a jump in $\Delta^{\text {perc }}(\Omega)$ if the location $z_{n}$ of a jump moves from $-\infty$ to $+\infty$ when $\Omega$ is increased infinitesimally (it does not lead to a jump if $z_{n}$ moves across 0 or 1 , as could be suspected at first sight, since the various contributions cancel in these cases). 
Fig. 1 The integrated density of states below the percolation threshold

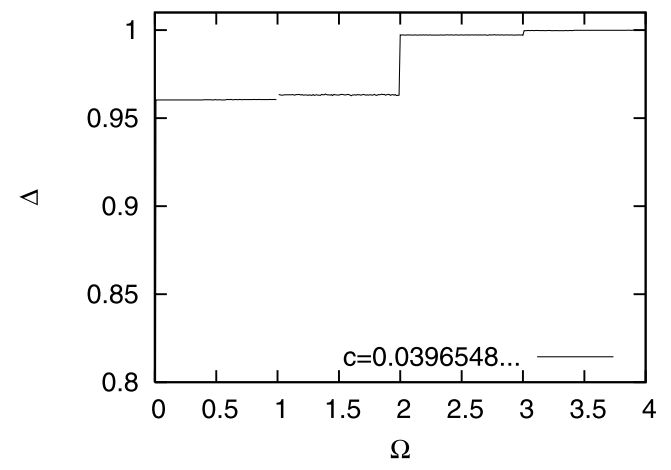

Equation (13) shows that $z_{n}$ can and does indeed pass $\infty$ as $\Omega$ is varied. While the peaks of the density of states of the finite clusters are located at those $\Omega$ for which $z_{n}=0$, the peaks for the percolating cluster are located where $z_{n}=\infty$. Since the zeros and the poles of $z_{n}$ necessarily alternate when $\Omega$ is varied, it follows that the peaks in the percolating cluster lie dense if the peaks in the finite clusters lie dense. In this sense there is no mobility edge in the percolating cluster since isolated and thus localised eigenvalues exist throughout the whole range of $0 \leq \Omega<\infty$.

Unfortunately, this argument only strictly holds if $b_{c, \Omega}(\lambda)$ is indeed continuous in $\Omega$. If it is not, cancellations might occur which could reduce (or even remove) the peaks from the percolating cluster. It is shown in Appendix B that indeed $b_{c, \Omega}(\lambda)$ is not continuous but the argument presented there also shows that a complete removal of peaks would seem an extremely fortuitous cancellation. In order to check these results we have performed population dynamics simulations of the integrated DSPC according to (17). We used population sizes of $10^{6}$ for Figs. 1, 2(a) and 3 and $8 \times 10^{6}$ for Fig. 2(b). The larger population size leads to a decreased statistical error which is important in Fig. 2(b) since the fluctuations could otherwise mask the small jump at $\Omega=1$. The fluctuations appear because the distributions $a_{c^{*}, \Omega}(\lambda)$ and $b_{c, \Omega}(\lambda)$ are only approximated by the finite populations.

An example of the integrated density of states below the percolation threshold is shown Fig. 1. The value of $c=0.0396548 \ldots$ used here is related by the symmetry of (2) to $c=2$ which appears in Fig. 2. This integrated density of states was not calculated from (18) (there is no percolating cluster below the threshold) but instead from (56) in Appendix A which holds for the total integrated density of states. Each small cluster contributes one null eigenvalue, hence the integrated density of states jumps from 0 to $\tau_{\text {tot }}(2 c)=1-c \approx 0.96$ (the number of clusters per site) at $\Omega=0$. For larger $\Omega$, steps are visible at $\Omega=1, \Omega=2$, and $\Omega=3$. However, it is clear from the solution in (11) and the coefficients $z_{k}$ that inbetween these locations the integrated density of states consists of infinitely many jumps which cannot be discerned on this scale (there is a jump whenever at least one of the $z_{k}$ is equal to 0 ).

Figure 2 shows the integrated DSPC directly at the critical point $c=\frac{1}{2}$ and deep inside the percolating regime at $c=2$. Some jumps are clearly observed for $c=\frac{1}{2}$, and they are located at the predicted positions. The most prominent ones occur at $\Omega=1$ where the coefficient $z=\frac{\Omega}{\Omega-1}=\infty$, or at $\Omega=\frac{3 \pm \sqrt{5}}{2}$ where $z=\frac{\Omega-\frac{\Omega}{\Omega-1}}{\Omega-1-\frac{\Omega}{\Omega-1}}=\infty$. For $c=2$ the integrated density of states in Fig. 2 looks smooth. This is, however, not the case as the close-up in Fig. 2(b) reveals. The jump at $\Omega=1$ which is very pronounced at $c=\frac{1}{2}$ is also present at $c=2$.

As another application of our method, we consider the asymptotic behaviour of the integrated density of states of the incipient percolating cluster at the percolation threshold 
Fig. 2 Figure (a) (top) shows the integrated density of states of the percolating cluster at $c=\frac{1}{2}$ and $c=2$. (b) (bottom) shows an enlargement of the curve for $c=2$ around $\Omega=1$

Fig. 3 Logarithmic plot of the integrated density of states of the percolating cluster at $c=\frac{1}{2}$
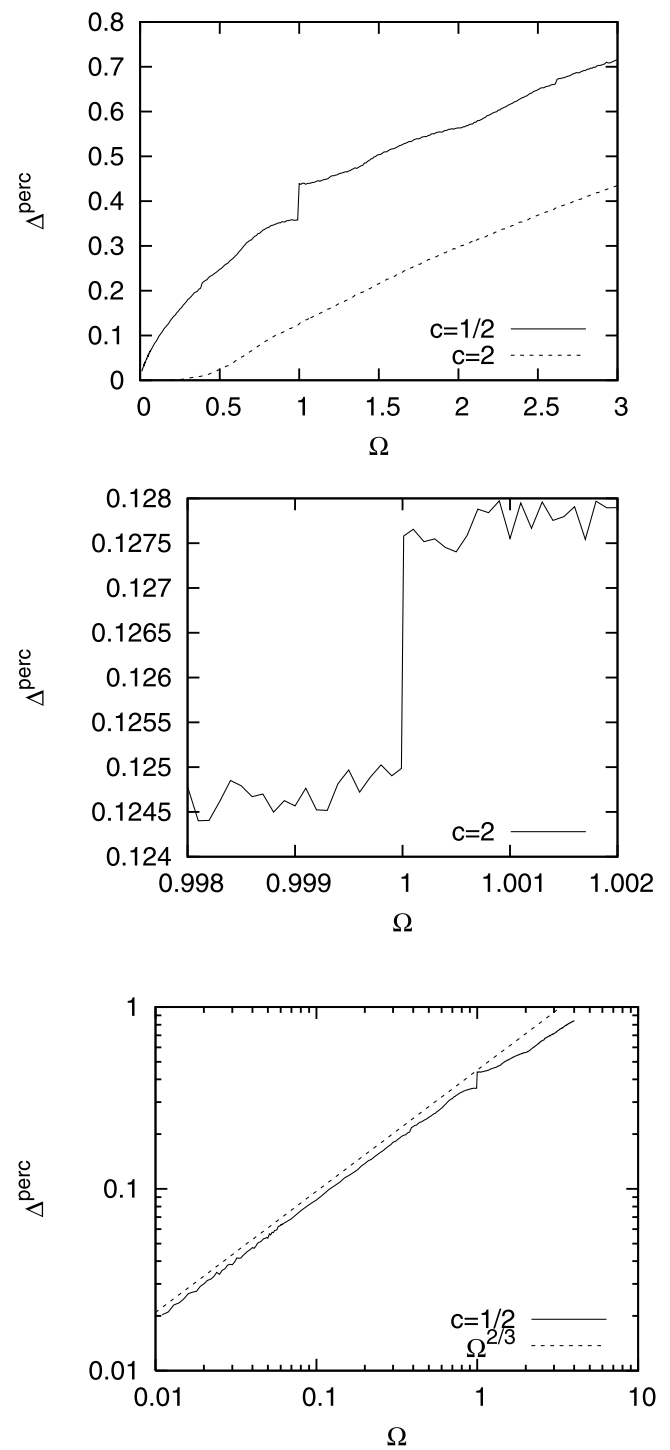

$c=\frac{1}{2}$. In Fig. 3 it is shown that for small $\Omega$ the integrated DSPC goes as $\Delta^{\text {perc }} \sim \Omega^{2 / 3}$. To the best of our knowledge, this has never been observed before in the literature. This result is in complete agreement with the Alexander-Orbach conjecture [15] according to which the integrated density of states of the incipient percolating cluster on finite dimensional lattices for small $\Omega$ goes with an exponent equal to one half of the spectral dimension of the giant cluster, which was suggested by Alexander and Orbach to be 4/3. The Alexander-Orbach conjecture is known to hold on hypercubic lattices of sufficiently high dimension for both percolation and oriented percolation [16, 17], but does not seem to hold in all dimensions. In addition it was shown for percolation on regular trees and the Bethe lattice [18, 19].

Acknowledgements Our work is based on ideas by Kurt Broderix who died on May 12, 2000. We thank Peter Müller and Reimer Kühn for valuable discussions. 
Open Access This article is distributed under the terms of the Creative Commons Attribution Noncommercial License which permits any noncommercial use, distribution, and reproduction in any medium, provided the original author(s) and source are credited.

\section{Appendix A: Integrated Density of States}

This section is intended to show how to derive the central equation (18). The density of states is given by

$$
D(\Omega)=\frac{1}{\pi} \lim _{N \rightarrow \infty \epsilon \searrow 0} \lim _{\Im} \Im(\Omega+i \epsilon)
$$

with

$$
\begin{aligned}
G(\Omega) & =\frac{1}{N} \operatorname{Tr} \overline{\frac{1}{\Omega-\Gamma}}=-\frac{2}{N} \frac{\partial}{\partial \Omega} \overline{\log Z(\Omega)} \\
& =-\lim _{n \rightarrow 0} \frac{2}{N n} \frac{\partial}{\partial \Omega} \log \overline{Z^{n}(\Omega)}
\end{aligned}
$$

and the "partition function" $Z(\Omega)=\operatorname{det}^{-1 / 2}(\Omega-\Gamma)$. The integrated density of states is therefore

$$
\Delta(\Omega)=\int_{0}^{\Omega} d \Omega^{\prime} D\left(\Omega^{\prime}\right)=\frac{1}{\pi} \lim _{N \rightarrow \infty \epsilon \searrow 0} \lim _{3} \Im(\Omega+i \epsilon)+\text { const. }
$$

with

$$
H(\Omega)=-\frac{2}{N} \overline{\log Z(\Omega)}=-\lim _{\epsilon \rightarrow 0} \lim _{n \rightarrow 0} \frac{2}{N n} \log \overline{Z^{n}(\Omega+i \epsilon)} .
$$

The averaged replicated partition function is given by $[3,10]$

$$
\begin{aligned}
\overline{Z^{n}(\Omega)}= & \exp (-N F(\Omega)) \\
= & \exp \left(-N\left(\frac{1}{2} \frac{\int d^{n} x e^{-\frac{i}{2} \Omega x^{2}+g^{2}(x)} g^{\Omega}(x)}{\int d^{n} x e^{-\frac{i}{2} \Omega x^{2}+g^{\Omega}(x)}}\right.\right. \\
& \left.\left.+c-\log \int \frac{d^{n} x}{(2 \pi)^{n / 2}} e^{-\frac{i}{2} \Omega x^{2}+g^{\Omega}(x)}\right)\right),
\end{aligned}
$$

where $g^{\Omega}(x)$ is the solution of the saddle point equation

$$
g^{\Omega}(x)=2 c \frac{\int d^{n} x^{\prime} e^{-\frac{i}{2} \Omega x^{\prime 2}+g^{\Omega}\left(x^{\prime}\right)-\frac{i}{2}\left(x-x^{\prime}\right)^{2}}}{\int d^{n} x^{\prime} e^{-\frac{i}{2} \Omega x^{\prime 2}+g^{\Omega}\left(x^{\prime}\right)}} .
$$

This equation becomes, assuming a replica symmetric $g^{\Omega}(x)=g^{\Omega}(|x|)=: g^{\Omega}(r)$,

$$
g^{\Omega}(r)=2 c \frac{(2 \pi)^{n / 2}}{S_{n}} \frac{\int d r^{\prime} r^{\prime n-1}\left(r r^{\prime}\right)^{1-\frac{n}{2}} J_{\frac{n}{2}-1}\left(r r^{\prime}\right) e^{-\frac{i}{2} \Omega r^{\prime 2}-\frac{i}{2} r^{2}-\frac{i}{2} r^{\prime 2}+g^{\Omega(}\left(r^{\prime}\right)}}{\int d r^{\prime} r^{\prime n-1} e^{-\frac{i}{2} \Omega r^{\prime 2}+g^{\Omega}\left(r^{\prime}\right)}},
$$

where $S_{n}=\frac{2 \pi^{n / 2}}{\Gamma\left(\frac{n}{2}\right)}$ is the surface area of the $n$-dimensional unit sphere. 
In order to calculate $H(\Omega)$, we need $\lim _{n \rightarrow 0} \frac{1}{n} F(\Omega)$, therefore we must expand the exponent in (23) in $n$. To this end, it will be necessary to calculate integrals of the form

$$
\int_{0}^{\infty} \frac{d r}{(2 \pi)^{n / 2}} r^{n-1} S_{n} f(r),
$$

where the function $f(r)$ is also a function of $n$. We assume that it can be expanded in $n$ as

$$
f(r)=\sum_{k=0}^{\infty} n^{k} f_{k}(r) .
$$

Then we have, for arbitrary $a>0$,

$$
\begin{aligned}
\int_{0}^{\infty} \frac{d r}{(2 \pi)^{n / 2}} r^{n-1} S_{n} f(r)= & \int_{0}^{\infty} \frac{d r}{(2 \pi)^{n / 2}} r^{n-1} S_{n} \sum_{k=0}^{\infty} n^{k} f_{k}(r) \\
= & \int_{0}^{\infty} \frac{d r}{(2 \pi)^{n / 2}} r^{n-1} S_{n} \sum_{k=0}^{\infty} n^{k}\left(f_{k}(r)-f_{k}(0) e^{-a r^{2} / 2}\right) \\
& +\int_{0}^{\infty} \frac{d r}{(2 \pi)^{n / 2}} r^{n-1} S_{n} \sum_{k=0}^{\infty} n^{k} f_{k}(0) e^{-a r^{2} / 2} \\
= & \frac{2}{\Gamma\left(\frac{n}{2}\right) 2^{n / 2}} \sum_{k=0}^{\infty} n^{k} \int_{0}^{\infty} \frac{d r}{r} e^{n \log r}\left(f_{k}(r)-f_{k}(0) e^{-a r^{2} / 2}\right) \\
& +\frac{2}{\Gamma\left(\frac{n}{2}\right) 2^{n / 2}} \sum_{k=0}^{\infty} n^{k} \int_{0}^{\infty} d r r^{n-1} e^{-a r^{2} / 2} f_{k}(0) \\
= & \frac{2}{\Gamma\left(\frac{n}{2}\right) 2^{n / 2}} \sum_{k=0}^{\infty} n^{k} \int_{0}^{\infty} \frac{d r}{r} e^{n \log r}\left(f_{k}(r)-f_{k}(0) e^{-a r^{2} / 2}\right) \\
& +\frac{2}{\Gamma\left(\frac{n}{2}\right) 2^{n / 2}} \sum_{k=0}^{\infty} n^{k} \frac{1}{2}\left(\frac{2}{a}\right)^{n / 2} \Gamma\left(\frac{n}{2}\right) f_{k}(0) \\
= & n \int_{0}^{\infty} \frac{d r}{r}\left(f_{0}(r)-f_{0}(0) e^{-a r^{2} / 2}\right)+\mathcal{O}\left(n^{2}\right) \\
& +f_{0}(0)-\frac{n}{2} f_{0}(0) \log a+n f_{1}(0)+\mathcal{O}\left(n^{2}\right) . \\
& \\
& \\
& \\
&
\end{aligned}
$$

The term $f_{k}(0) e^{-a r^{2} / 2}$ involving the constant $a$ has been introduced to avoid problems with the divergence of the integrals at the lower boundary. The result is valid for any positive value of $a$ such that we may later choose $a$ to our convenience, if necessary. Note that this result involves $f_{1}(0)$, which stems from the next-to-leading order in the $n$-expansion. For the ordinary calculation of $G(\Omega)$ such terms do not appear. Here, for $H(\Omega)$, however, they will turn out to be crucial.

We can now calculate the integrals in (23). For the numerator of the first term of $F(\Omega)$ we have

$$
f(r)=e^{-\frac{i}{2} \Omega r^{2}+g^{\Omega}(r)} g^{\Omega}(r)
$$




$$
\begin{aligned}
& =e^{-\frac{i}{2} \Omega r^{2}+g_{0}^{\Omega}(r)}\left(1+n g_{1}^{\Omega}(r)+\cdots\right)\left(g_{0}^{\Omega}(r)+n g_{1}^{\Omega}(r)+\cdots\right) \\
& =e^{-\frac{i}{2} \Omega r^{2}+g_{0}^{\Omega}(r)}\left(g_{0}^{\Omega}(r)+n g_{1}^{\Omega}(r)\left(1+g_{0}^{\Omega}(r)\right)+\mathcal{O}\left(n^{2}\right)\right)
\end{aligned}
$$

expanding $g^{\Omega}(r)=\sum_{k=0}^{\infty} n^{k} g_{k}^{\Omega}(r)$. Therefore

$$
\begin{aligned}
& \int \frac{d^{n} x}{(2 \pi)^{n / 2}} e^{-\frac{i}{2} \Omega x^{2}+g^{\Omega}(x)} g^{\Omega}(x) \\
& =f_{0}(0)+n\left[\int_{0}^{\infty} \frac{d r}{r}\left(f_{0}(r)-f_{0}(0) e^{-a r^{2} / 2}\right)-\frac{1}{2} f_{0}(0) \log a+f_{1}(0)\right]+\mathcal{O}\left(n^{2}\right) \\
& =e^{g_{0}^{\Omega}(0)}\left(g_{0}^{\Omega}(0)+n\left[\int_{0}^{\infty} \frac{d r}{r}\left(e^{-\frac{i}{2} \Omega r^{2}+g_{0}^{\Omega(}(r)-g_{0}^{\Omega}(0)} g_{0}^{\Omega}(r)-g_{0}^{\Omega}(0) e^{-a r^{2} / 2}\right)\right.\right. \\
& \left.\left.\quad-\frac{1}{2} \log a+g_{1}^{\Omega}(0)\left(1+g_{0}^{\Omega}(0)\right)\right]\right)+\mathcal{O}\left(n^{2}\right)
\end{aligned}
$$

For the denominator we get likewise

$$
\begin{aligned}
f(r) & =e^{-\frac{i}{2} \Omega r^{2}+g^{\Omega}(r)} \\
& =e^{-\frac{i}{2} \Omega r^{2}+g_{0}^{\Omega}(r)}\left(1+n g_{1}^{\Omega}(r)+\mathcal{O}\left(n^{2}\right)\right)
\end{aligned}
$$

and thus

$$
\begin{aligned}
\int \frac{d^{n} x}{(2 \pi)^{n / 2}} e^{-\frac{i}{2} \Omega x^{2}+g^{\Omega}(x)}= & e^{g_{0}^{\Omega}(0)}\left(1+n\left[\int_{0}^{\infty} \frac{d r}{r}\left(e^{-\frac{i}{2} \Omega r^{2}+g_{0}^{\Omega}(r)-g_{0}^{\Omega}(0)}-e^{-a r^{2} / 2}\right)\right.\right. \\
& \left.\left.-\frac{1}{2} \log a+g_{1}^{\Omega}(0)\right]\right)+\mathcal{O}\left(n^{2}\right) .
\end{aligned}
$$

Using these results, we get the following expression for $F(\Omega)$ :

$F(\Omega)$

$$
\begin{aligned}
= & \frac{1}{2}\left(g_{0}^{\Omega}(0)+n\left[\int_{0}^{\infty} \frac{d r}{r}\left(e^{-\frac{i}{2} \Omega r^{2}+g_{0}^{\Omega}(r)-g_{0}^{\Omega}(0)} g_{0}^{\Omega}(r)-g_{0}^{\Omega}(0) e^{-a r^{2} / 2}\right)\right.\right. \\
& \left.\left.-\frac{1}{2} \log a+g_{1}^{\Omega}(0)\left(1+g_{0}^{\Omega}(0)\right)\right]+\mathcal{O}\left(n^{2}\right)\right) \\
& \times\left(1+n\left[\int_{0}^{\infty} \frac{d r}{r}\left(e^{-\frac{i}{2} \Omega r^{2}+g_{0}^{\Omega}(r)-g_{0}^{\Omega}(0)}-e^{-a r^{2} / 2}\right)-\frac{1}{2} \log a+g_{1}^{\Omega}(0)\right]+\mathcal{O}\left(n^{2}\right)\right) \\
& +c-g_{0}^{\Omega}-\log \left(1+n\left[\int_{0}^{\infty} \frac{d r}{r}\left(e^{-\frac{i}{2} \Omega r^{2}+g_{0}^{\Omega}(r)-g_{0}^{\Omega}(0)}-e^{-a r^{2} / 2}\right)-\frac{1}{2} \log a+g_{1}^{\Omega}(0)\right]\right. \\
& \left.+\mathcal{O}\left(n^{2}\right)\right) \\
= & n\left(-\frac{1}{2} g_{1}^{\Omega}(0)+\frac{1}{2} \log a-(c+1) \int_{0}^{\infty} \frac{d r}{r}\left(e^{-\frac{i}{2} \Omega r^{2}+g_{0}^{\Omega}(r)-2 c}-e^{-a r^{2} / 2}\right)\right. \\
& \left.+\int_{0}^{\infty} \frac{d r}{r}\left(e^{-\frac{i}{2} \Omega r^{2}+g_{0}^{\Omega}(r)-2 c} g_{0}^{\Omega}(r)-2 c e^{-a r^{2} / 2}\right)\right)+\mathcal{O}\left(n^{2}\right),
\end{aligned}
$$

employing the known fact that $g_{0}^{\Omega}(0)=2 c$. 
We see that the missing piece of information is $g_{1}^{\Omega}(0)$. We must go back to (24) and use it to calculate $g_{1}^{\Omega}(0)$. When we take the limit $r \rightarrow 0$, then $\left(r r^{\prime}\right)^{1-n / 2} J_{\frac{n}{2}-1}\left(r r^{\prime}\right) \rightarrow \frac{S_{n}}{(2 \pi)^{n / 2}}$, so the equation becomes

$$
\begin{aligned}
& g_{0}^{\Omega}(0)+n g_{1}^{\Omega}(0)+\cdots \\
& =2 c \frac{\int_{0}^{\infty} \frac{d r}{(2 \pi)^{n / 2}} S_{n} r^{n-1} e^{-\frac{i}{2}(\Omega+1) r^{2}+g_{0}^{\Omega}(r)-g_{0}^{\Omega}(0)}\left(1+n g_{1}^{\Omega}(r)+\cdots\right)}{\int_{0}^{\infty} \frac{d r}{(2 \pi)^{n / 2}} S_{n} r^{n-1} e^{-\frac{i}{2} \Omega r^{2}+g_{0}^{\Omega}(r)-g_{0}^{\Omega}(0)}\left(1+n g_{1}^{\Omega}(r)+\cdots\right)} \\
& =2 c\left(1+n \int_{0}^{\infty} \frac{d r}{r}\left(e^{-\frac{i}{2}(\Omega+1) r^{2}+g_{0}^{\Omega}(r)-g_{0}^{\Omega}(0)}-e^{-\frac{i}{2} \Omega r^{2}+g_{0}^{\Omega}(r)-g_{0}^{\Omega}(0)}\right)\right)+\mathcal{O}\left(n^{2}\right),
\end{aligned}
$$

such that

$$
g_{1}^{\Omega}(0)=2 c \int_{0}^{\infty} \frac{d r}{r}\left(e^{-\frac{i}{2}(\Omega+1) r^{2}+g_{0}^{\Omega}(r)-g_{0}^{\Omega}(0)}-e^{-\frac{i}{2} \Omega r^{2}+g_{0}^{\Omega}(r)-g_{0}^{\Omega}(0)}\right) .
$$

Inserting this into (39) gives

$$
\begin{aligned}
F(\Omega)= & n\left(\frac{1}{2} \log a-c \int_{0}^{\infty} \frac{d r}{r}\left(e^{-\frac{i}{2}(\Omega+1) r^{2}+g_{0}^{\Omega}(r)-2 c}-e^{-a r^{2} / 2}\right)\right. \\
& -\int_{0}^{\infty} \frac{d r}{r}\left(e^{-\frac{i}{2} \Omega r^{2}+g_{0}^{\Omega}(r)-2 c}-e^{-a r^{2} / 2}\right) \\
& \left.+\int_{0}^{\infty} \frac{d r}{r}\left(e^{-\frac{i}{2} \Omega r^{2}+g_{0}^{\Omega}(r)-2 c} g_{0}^{\Omega}(r)-2 c e^{-a r^{2} / 2}\right)\right) .
\end{aligned}
$$

This is the final form of the "free energy" as it has been fully reduced to the quantity $g_{0}^{\Omega}(r)$. Remember that the parameter $a>0$ is arbitrary and will later disappear anyway upon taking the imaginary value.

Now we need to express this in terms of the probability distribution $a_{c, \Omega}(\lambda)$, in terms of which $g_{0}^{\Omega}(r)$ can be written as

$$
g_{0}^{\Omega}(r)=2 c \int_{-\infty}^{\infty} d a_{c, \Omega}(\lambda) e^{-\frac{i}{2} \lambda r^{2}} .
$$

We would like to express $e^{-\frac{i}{2} \Omega r^{2}+g_{0}^{\Omega}(r)}$ in a simple way using $a_{c, \Omega}(\lambda)$, too. This is possible via the following observation: The integral equation (24) (at $n=0$ ) can be written as

$$
\begin{aligned}
g_{0}^{\Omega}(r) & =2 c e^{-\frac{i}{2} r^{2}}-2 c e^{-\frac{i}{2} r^{2}} \int_{0}^{\infty} d r^{\prime} r J_{1}\left(r r^{\prime}\right) e^{-\frac{i}{2}(\Omega+1) r^{\prime 2}+g_{0}^{\Omega}\left(r^{\prime}\right)-2 c} \\
& =2 c e^{-\frac{i}{2} r^{2}}-2 c\left(K e^{-\frac{i}{2}(\Omega+1) r^{\prime 2}+g_{0}^{\Omega}\left(r^{\prime}\right)-2 c}\right)(r),
\end{aligned}
$$

where $K \cdot$ is the linear integral operator

$$
(K f)(r)=e^{-\frac{i}{2} r^{2}} \int_{0}^{\infty} d r^{\prime} r J_{1}\left(r r^{\prime}\right) f\left(r^{\prime}\right) .
$$

Then

$$
e^{-\frac{i}{2} \Omega r^{2}+g_{0}^{\Omega}(r)-2 c}=\left(K^{-1}\left(\frac{1}{2 c} g_{0}^{\Omega}\left(r^{\prime}\right)-e^{-\frac{i}{2} r^{\prime 2}}\right)\right)(r)
$$




$$
\begin{aligned}
& =\left(K^{-1} \int_{-\infty}^{\infty} d a_{c, \Omega}(\lambda)\left(e^{-\frac{i}{2} \lambda r^{\prime 2}}-e^{-\frac{i}{2} r^{\prime 2}}\right)\right)(r) \\
& =\left(K^{-1} \int_{-\infty}^{\infty} d a_{c, \Omega}(\lambda)\left(K e^{-\frac{i}{2} \frac{\lambda}{1-\lambda} r^{\prime \prime 2}}\right)\left(r^{\prime}\right)\right)(r) \\
& =\int_{-\infty}^{\infty} d a_{c, \Omega}(\lambda) e^{-\frac{i}{2} \frac{\lambda}{1-\lambda} r^{2}} .
\end{aligned}
$$

Similarly,

$$
e^{-\frac{i}{2}(\Omega+1) r^{2}+g_{0}^{\Omega}(r)-2 c}=\int_{-\infty}^{\infty} d a_{c, \Omega}(\lambda) e^{-\frac{i}{2}\left(\frac{z}{1-z}+1\right) r^{2}} .
$$

Let's look at one of the integrals, e.g.

$$
\begin{aligned}
& \int_{0}^{\infty} \frac{d r}{r}\left(e^{-\frac{i}{2} \Omega r^{2}+g_{0}^{\Omega}(r)-2 c}-e^{-a r^{2} / 2}\right) \\
& \quad=\int_{0}^{\infty} \frac{d r}{r}\left(\int_{-\infty}^{\infty} d a_{c, \Omega}(\lambda) e^{-\frac{i}{2}\left(\frac{\lambda}{1-\lambda}+1\right) r^{2}}-e^{-a r^{2} / 2}\right)
\end{aligned}
$$

Ultimately, we will only be interested in the imaginary part of this. We can then forget about the term containing $a$ as it is real. Then

$$
\Im \int_{0}^{\infty} \frac{d r}{r} e^{-\frac{i}{2} \Omega r^{2}+g_{0}^{\Omega}(r)-2 c}=\int_{0}^{\infty} \frac{d r}{r} \int_{-\infty}^{\infty} d a_{c, \Omega}(\lambda) \sin \left(-\frac{1}{2} \frac{\lambda}{1-\lambda} r^{2}\right) .
$$

Exchanging the order of integration and carrying out the integral over $r$, one gets

$$
\Im \int_{0}^{\infty} \frac{d r}{r} e^{-\frac{i}{2} \Omega r^{2}+g_{0}^{\Omega}(r)-2 c}=\int_{-\infty}^{\infty} d a_{c, \Omega}(\lambda) \frac{\pi}{4} \operatorname{sgn}\left(\frac{\lambda}{\lambda-1}\right) .
$$

Similar results hold for the other two types of integral.

Finally, we get for the integrated density of states

$$
\begin{aligned}
\Delta(\Omega)= & \frac{1}{2}\left(\int_{-\infty}^{\infty} d a_{c, \Omega}(\lambda) \operatorname{sgn}\left(\frac{\lambda}{\lambda-1}\right)+c \int_{-\infty}^{\infty} d a_{c, \Omega}(\lambda) \operatorname{sgn}\left(\frac{\lambda}{\lambda-1}-1\right)\right. \\
& \left.-c \int_{-\infty}^{\infty} d a_{c, \Omega}(\lambda) d a_{c, \Omega}\left(\lambda^{\prime}\right) \operatorname{sgn}\left(\frac{\lambda}{\lambda-1}-\lambda^{\prime}\right)\right)+\frac{1}{2} .
\end{aligned}
$$

The constant of integration, i.e. the trailing $\frac{1}{2}$ in the above equation, can be calculated as follows. For $c<\frac{1}{2}$ it is obvious from the definition of $z_{n}$ that in the limit $\Omega \rightarrow-\infty$ all $z_{n}$ tend to 1 from below. The evaluation of the integrals in (56) is then easy and gives $-\frac{1}{2}$, thus the constant of integration must be $\frac{1}{2}$ since $D(-\infty)=0$. For $c \geq \frac{1}{2}$ a similar argument holds, as the main body of the paper shows that in the limit that all $z_{n} \uparrow 1, \lambda \uparrow 1$ as well. Equation (56) can thus be evaluated as above, and the constant is again $\frac{1}{2}$.

Equation (56) is the equation for the total integrated density of states. We are interested in the density of states of the percolating cluster, which can be obtained from this by subtracting the part from the finite clusters. This can be done by evaluating (56) both at $c \geq \frac{1}{2}$ and at the corresponding $c^{*} \leq \frac{1}{2}$ and subtracting the latter from the former (suitably weighted). 
Decomposing $a_{c, \Omega}(\lambda)$ into the two parts pertaining to the finite clusters at $c^{*} \leq \frac{1}{2}$ and the percolating cluster at $c \geq \frac{1}{2}$,

$$
2 c a_{c, \Omega}(\lambda)=2 c^{*} a_{c^{*}, \Omega}(\lambda)+\left(2 c-2 c^{*}\right) b_{c, \Omega}(\lambda),
$$

then yields (18) from the main text.

\section{Appendix B: Non-Continuity of $b_{c, \Omega}(\lambda)$}

In this section we discuss the question whether the population density $b_{c, \Omega}(\lambda)$ is continuous as a function of $\Omega$ or not. In Sect. 4 a simple argument was given to show that continuity implies that localised eigenvalues of the percolating cluster lie dense. Here we will show that $b_{c, \Omega}(\lambda)$ is in fact not continuous. Nevertheless we conjecture that the localised eigenvalues still lie dense since the cancellations that would have to occur to produce a gap appear extremely fortuitous.

In order to judge whether $b_{c, \Omega}(\lambda)$ is continuous, we examine the update rule $\lambda=1-$ $\frac{1}{\frac{1}{1-z}+\sum_{i=1}^{M} \lambda_{i}}$ contained in (17). Suppose $\Omega$ is such that it lies exactly at a pole of, say, $z_{n}$ (so $\left.z_{n}=\infty\right)$, then it follows from (12)-(13) that there are some $n^{\prime}$ with $z_{n^{\prime}}=1$. In this case the update rule shows that with finite probability (namely the probability that one of these $n^{\prime}$ is picked) $\lambda=1$ is inserted into the population. Hence $b_{c, \Omega}(\lambda)$ has a jump at $\lambda=1$ as a function of $\lambda$. This implies that other discrete values of $\lambda$ are also present with finite probability. Take for example the case that $z=z_{n}=\infty$ is picked (which happens with finite probability), and that only $\lambda_{i}=1$ are chosen (the probability of which is finite as well, according to our reasoning above), then obviously $\lambda=1-\frac{1}{M}$ for $M=1,2, \ldots$ are all generated with finite probability. Among these is $\lambda=0$. But when $\lambda=0$ is present with finite probability, then $\lambda=z_{n^{\prime \prime}}$ has finite probability for any $n^{\prime \prime}$. Thus we see that all $z_{n^{\prime \prime}}$ from the finite clusters are reproduced in the weight function $b_{c, \Omega}(\lambda)$ of the percolating cluster, although with different weights. Of course, as mentioned above, this is only true when $\Omega$ lies at a pole of some $z_{n}$. However, it shows that $b_{c, \Omega}(\lambda)$ is not continuous as a function of $\Omega$ since (at least) $z_{n}$ moves across $\infty$ as $\Omega$ is varied across the pole of $z_{n}$ such that a finite amount of weight is shifted from $-\infty$ to $+\infty$ in the $\lambda$ population, resulting in a jump in $b_{c, \Omega}(\lambda)$ for any fixed $\lambda$.

\section{References}

1. Mehta, M.: Random Matrices. Academic Press, Boston (1991)

2. Guhr, T., Müller-Groeling, A., Weidenmüller, H.A.: Phys. Rep. 299, 190 (1998)

3. Bray, A.J., Rodgers, G.J.: Phys. Rev. B 38, 11461 (1988)

4. Fyodorov, Y.V., Mirlin, A.D.: J. Phys. A, Math. Gen. 24, 2219 (1991)

5. Khorunzhy, O., Shcherbina, M., Vengerovsky, V.: J. Math. Phys. 45, 1648 (1994)

6. Khorunzhy, O., Kirsch, W., Müller, P.: Ann. Appl. Probab. 16, 295 (2006)

7. Bauer, M., Golinelli, O.: J. Stat. Phys. 103, 301 (2001)

8. Biroli, G., Monasson, R.: J. Phys. A 32, L255 (1999)

9. Kühn, R.: J. Phys. A, Math. Gen. 41, 295002 (2008)

10. Broderix, K., Aspelmeier, T., Hartmann, A.K., Zippelius, A.: Phys. Rev. E 64, 021404 (2001)

11. Müller, P., Stollmann, P.: arXiv:1002.5000

12. Erdős, P., Rényi, A.: Magyar Tud. Akad. Mat. Kut. Int. Kőzl. 5, 17 (1960). Reprinted in: Spencer, J. (ed.): The Art of Counting. MIT Press, Cambridge (1973)

13. Reed, M., Simon, B.: Methods of Modern Mathematical Physics, vol. 1: Functional Analysis. Academic Press, New York (1972)

14. Chayes, J.T., Chayes, L., Franz, J.R., Sethna, J.P.: J. Phys. A, Math. Gen. 19, L1173 (1986) 
15. Alexander, S., Orbach, R.: J. Phys. Lett. (Paris) 43, L625 (1982)

16. Barlow, M.T., Járai, A.A., Kumagai, T., Slade, G.: Commun. Math. Phys. 278, 385 (2008)

17. Kozma, G., Nachmias, A.: Invent. Math. 178, 635 (2009)

18. Kesten, H.: Ann. Inst. H. Poincaré Probab. Stat. 22, 425 (1986)

19. Barlow, M.T., Kumagai, T.: Ill. J. Math. 50, 33 (2006) 